\title{
PERCEPCIONES Y PREFERENCIAS DE ESTUDIANTES DE INGLÉS EN SECUNDARIA SOBRE LA DEVOLUCIÓN DOCENTE ESCRITA
}

\author{
HIGH-SCHOOL EFL STUDENTS' PERCEPTIONS AND \\ PREFERENCES ON WRITTEN CORRECTIVE FEEDBACK
}

\author{
Eliana Berardo* \\ Pedro Luchini*
}

Rec.: 09-09-2021. Acept.: 19-10-2021. Publ.: 20-12-2021

DOI: https://doi.org/10.29035/ucmaule.61.11

\section{RESUMEN}

En la actualidad, las discusiones teóricas acerca de la devolución docente han cambiado de eje. Generalmente, los cuestionamientos acerca de la devolución docente escrita rondan en función de su cantidad y naturaleza como aspectos determinantes de efectividad. Además de la mirada psicolingüística sobre la devolución docente, informada por teorías de adquisición de lenguas, es importante considerar el aspecto afectivo. La interpretación de la devolución que los estudiantes realicen estará determinada, en gran medida, por sus expectativas. Este estudio se propone conocer las percepciones de dos grupos de estudiantes de inglés como L2 en el nivel secundario de la ciudad de Mar del Plata (Argentina) acerca de sus preferencias en cuanto a cantidad y tipo de devolución docente. Los participantes, en cada grupo, poseían diferentes niveles de competencia lingüística en L2, lo que permitió comparar/contrastar resultados y determinar, en qué medida, estas diferencias incidieron en sus percepciones y preferencias acerca de la devolución docente escrita recibida. Los resultados mostraron que ambos grupos de estudiantes reconocieron el valor de la devolución docente como herramienta de aprendizaje, prefiriendo recibir devolución no focalizada. Asimismo, los estudiantes más avanzados resultaron ser más receptivos frente al uso de preguntas e imperativos en los comentarios de los docentes.

\footnotetext{
* https://orcid.org/0000-0002-0030-4261 Universidad Nacional de Mar del Plata

* https://orcid.org/0000-0002-7692-8361 Universidad Nacional de Mar del Plata
} 
Berardo, E. \& Luchini, P. (2021). Percepciones y preferencias de estudiantes de inglés en secundaria sobre la devolución docente escrita. UCMaule, 61, julio-diciembre, 11-33. https://doi.org/10.29035/ ucmaule.61.11

Palabras clave: devolución docente, preferencias, percepciones, inglés, escritura

\section{ABSTRACT}

There has been a shift in focus in the theoretical discussions on written corrective feedback. Nowadays, it is less common to challenge its overall effectiveness. Researchers tend to focus on the amount and the type of feedback provided, as these aspects are believed to determine its effectiveness. In addition to the psycholinguistic perspectives that take into account different language acquisition theories, the affective aspect of student-teacher interaction should also be considered. The way students interpret written corrective feedback will be determined, to a great extent, by their expectations. The present study looks into the preferences of two groups of high-school students of English as a foreign language in Mar del Plata, Argentina, as to the quantity and quality of written corrective feedback they receive. These two groups of students had different levels of English competence. This helped determine whether these differences in L2 command had an impact on their preferences and expectations as regards written corrective feedback. The results showed that both groups recognized the value of teacher feedback as a learning tool, and preferred to receive non-targeted feedback. Likewise, the more advanced students turned out to be more receptive to the use of questions and imperatives in their teachers' comments.

Key words: feedback, preferences, perceptions, English, writing

\section{INTRODUCCIÓN}

La devolución docente en las tareas de escritura constituye una de las principales vías de interacción entre docentes y estudiantes. Las teorías de adquisición de la lengua que se basan en la interacción, tales como el modelo de Gass (2018) y la teoría del Sistema Adaptativo Complejo (Beckner et al., 2009), destacan el rol que tiene la devolución docente en el desarrollo de la interlengua del sujeto que aprende. En un contexto donde se propone la enseñanza del inglés como lengua extranjera (de ahora en adelante, L2) dentro de un contexto comunicativo, los ejercicios mecánicos enfocados en determinadas estructuras gramaticales ya no son el único medio de práctica y evaluación de la adquisición de estructuras nuevas. Las tareas de escritura contextualizadas son una de las tantas oportunidades que los estudiantes tienen de usar la lengua de forma comunicativa y de probar las hipótesis que hayan formulado 
Berardo, E. \& Luchini, P. (2021). Percepciones y preferencias de estudiantes de inglés en secundaria sobre la devolución docente escrita. UCMaule, 67, julio-diciembre, 11-33. https://doi.org/10.29035/ ucmaule.61.11

sobre el uso de las estructuras que están incorporando en su interlengua. La devolución docente como respuesta a estas tareas brindará evidencia para la reestructuración de la interlengua, en el caso de ser necesario.

Hace algunas décadas, la efectividad de la devolución docente, principalmente sobre aspectos gramaticales, fue puesta en duda. Hoy en día, se sostiene la idea de que la devolución docente es efectiva, y que su grado de efectividad podría variar de acuerdo a la cantidad y al tipo de devolución. El acto de brindar y recibir devolución es un acto comunicativo en sí mismo, por tanto, no debe perderse de vista su nivel pragmático. Los docentes deben tomar decisiones sobre la cantidad y el tipo de comentarios a realizar, y estas decisiones determinarán el efecto que tendrá en los estudiantes que los reciban. Por eso es que la devolución docente debe repensarse, replantearse y adaptarse para que se eviten efectos indeseados en el plano afectivo y lograr que la devolución pueda ser beneficiosa. Los hallazgos y conclusiones de este estudio brindarán datos a docentes de L2 para conocer qué cantidad y qué tipo de devolución esperan los estudiantes $y$, de este modo, poder tomar decisiones fundamentadas sobre estos aspectos a fin de que la comunicación con sus estudiantes sea más efectiva.

\section{La devolución docente}

Según Burke y Pieterick (2010), la devolución docente puede definirse como "un sistema de guía" en el que se le brinda información al estudiante sobre su evolución en relación al objetivo final con la intención de que pueda realizar las modificaciones necesarias para alcanzarlo satisfactoriamente. Este sistema de guía se conforma por medio de diversos tipos de comentarios e intervenciones. En el caso de la devolución docente escrita, Ellis (2009), propone una detallada taxonomía de la clase de comentarios posibles. Entre los criterios que sigue para organizar esta clasificación se encuentran el tipo de información que se le brinda al estudiante y el foco del comentario. Al encontrar un error o un aspecto a mejorar en el texto del estudiante, el docente puede elegir dar una alternativa correcta (devolución directa), evidenciar la existencia y ubicación del error sin dar una alternativa (devolución indirecta) o evidenciar la existencia de un error sin dar la respuesta correcta, pero incluyendo un indicio metalingüístico (devolución metalingüística). Por otro lado, la devolución docente puede ser focalizada o no focalizada. Por devolución focalizada se entiende aquella en la que se destacan sólo algunos errores teniendo en cuenta alguna categoría en particular, mientras que en la devolución no focalizada se trata de corregir todos los errores encontrados en un texto, sin una categorización preconcebida en la que centrarse (Ferris, 2002). 
Berardo, E. \& Luchini, P. (2021). Percepciones y preferencias de estudiantes de inglés en secundaria sobre la devolución docente escrita. UCMaule, 67, julio-diciembre, 11-33. https://doi.org/10.29035/ ucmaule.61.11

\section{El rol del feedback en la adquisición y el desarrollo de la lengua: aspectos teóricos}

El feedback tiene un rol fundamental en teorías que explican la adquisición de lenguas mediante la interacción, como el modelo de Gass (2018). Prometiendo relacionar teorías generativistas con interaccionistas, Gass explica la adquisición de segundas lenguas mediante un modelo propio que detalla cómo el aprendiz pasa del input (exposición a la segunda lengua) al output (producción propia usando la segunda lengua). Este modelo, entonces, consiste en distintas etapas relacionadas que llevan del input al output:

1. Input comprendido: Gass define "comprensión" en un sentido amplio: "representa un continuo de posibilidades que van desde la semántica hasta el análisis estructural detallado" (2018, p. 5). El nivel de análisis del input es lo que va a determinar en qué medida este se va a convertir en intake (entrada).

2. Intake: es la incorporación del input en el sistema de interlengua del aprendiz. Esta incorporación está mediada por las estructuras previamente internalizadas en el sistema. En este momento del proceso es donde se dan las generalizaciones y la fosilización.

3. Integración: la información lingüística que pasa por el proceso de intake puede ser integrada al sistema de interlengua del aprendiz o no. En el caso de que sea integrada, puede estar confirmando o refutando hipótesis creadas por el aprendiz sobre el funcionamiento de la lengua y quizás, a su vez, esto llegue a modificar su uso. Por otro lado, si la información no es integrada, puede ser que sólo se almacene en la memoria hasta encontrar más evidencia para formar hipótesis más claras, o que simplemente se deseche esa información por no haberla podido analizar a un nivel significativo.

4. Output: Gass define a esta etapa como una "manifestación explícita del proceso" (2018, p. 7). Sin embargo, no es sólo el resultado de la adquisición de la lengua, sino que es parte activa del proceso al ser el mecanismo de prueba de las hipótesis creadas en el proceso de incorporación de nueva información lingüística en la interlengua. En esta última etapa, el feedback que reciba el aprendiz va a servir como nueva evidencia, nuevo input, que pueda confirmar o refutar las hipótesis vigentes en su interlengua. 
Berardo, E. \& Luchini, P. (2021). Percepciones y preferencias de estudiantes de inglés en secundaria sobre la devolución docente escrita. UCMaule, 67, julio-diciembre, 11-33. https://doi.org/10.29035/ ucmaule.61.11

Para que el feedback produzca un efecto de reestructuración de la interlengua es necesario que sea input susceptible de ser comprendido por el aprendiz, pero también es necesario que existan otras instancias en las que se provea evidencia sobre el funcionamiento de esa misma característica de la lengua.

Esta última consideración se relaciona con el debate que se inició décadas atrás acerca de la efectividad del feedback con respecto al desarrollo de la interlengua. Mientras que algunos autores, en el pasado, indicaron que el feedback tiene un efecto limitado o nulo sobre la interlengua de los aprendices (Truscott, 1996), diversos estudios han demostrado lo contrario e incluso afirman que el feedback es de suma importancia para aprendices adultos que son menos propensos a la adquisición incidental de estructuras y cuando las estructuras estudiadas no son utilizadas con frecuencia, o no son lo suficientemente salientes como para ser notadas sin asistencia (Ellis et al., 2008).

La teoría del Sistema Adaptativo Complejo (Beckner et al., 2009) también pone de relieve la importancia del feedback, proponiendo que el desarrollo de la lengua no puede darse en el hablante o el aprendiz aislado del resto de la comunidad. El desarrollo no se produce como el mero resultado de procesos cognitivos, sino que la lengua se moldea como resultado de numerosos factores, entre los cuales se destaca la interacción. La lengua en sí, es el resultado de la interacción de los distintos idiolectos. La evidencia de uso de la lengua, o el input, tiene también la capacidad de influir en el desarrollo de los idiolectos, o la interlengua del aprendiz, ya que son sistemas abiertos. En el aula de lenguas extranjeras, el feedback es una instancia más de interacción y de evidencia que puede llegar a producir este desarrollo.

\section{Evidencia sobre la efectividad de la devolución docente escrita}

Algunos autores, como Truscott (1996; 1999; 2004; 2007), han cuestionado la efectividad de esta técnica para ayudar a los estudiantes a desarrollar su competencia lingüística. Truscott propone a los docentes abandonar la corrección sobre aspectos gramaticales dado que este tipo de práctica no tendrá un efecto positivo a largo plazo siendo que los estudiantes desarrollan su interlengua a partir de conocimientos implícitos (Ellis, 2015), adquiridos mediante la exposición a la lengua. Por otro lado, Ferris (1997; 1999; 2002) argumenta que la devolución docente sobre aspectos gramaticales no debe ser abandonada — siempre y cuando sea indirecta— para que los estudiantes se involucren al intentar encontrar la alternativa correcta para resolver el problema. Sin embargo, reconoció la necesidad de seguir investigando para comprobar la veracidad de estos supuestos. 
Berardo, E. \& Luchini, P. (2021). Percepciones y preferencias de estudiantes de inglés en secundaria sobre la devolución docente escrita. UCMaule, 61, julio-diciembre, 11-33. https://doi.org/10.29035/ ucmaule.61.11

Otros investigadores retomaron este debate en los últimos años, aportando datos que indicarían que la devolución docente sobre gramática es o bien beneficiosa para la adquisición de un idioma o, al menos, inocua. Bitchener (2008) estudió el efecto de la devolución docente en el uso del sistema de artículos a, an y the. Concluyó que la devolución docente fue efectiva con resultados satisfactorios en el largo plazo y en nuevas tareas de escritura. Años más tarde, Bitchener (2012) propuso que la efectividad de la devolución docente estará sujeta a lo propuesto en teorías del procesamiento de la información. Las implicancias pedagógicas que se desprenden de esa propuesta son varias. Los estudiantes deben recibir la devolución docente y concentrarse en interpretarla. Asimismo, deben notar el contraste entre lo que ellos escribieron y la información que proveen los docentes y ser capaces de recuperar la información necesaria desde su memoria a largo plazo. Finalmente, deben estar listos para adquirir la forma señalada, de acuerdo al nivel de complejidad en el que se encuentra desarrollada su interlengua.

Ellis et al. (2008) analizaron la devolución docente focalizada y no focalizada en un estudio cuasi-experimental con estudiantes de ILE japoneses. Dividieron a los participantes en tres grupos y les dieron un tipo de devolución docente diferente a dos de ellos, mientras que el otro no recibió devolución. Se enfocaron, al igual que Bitchener (2008), en la precisión del uso del sistema de artículos a, an y the. Mediante la utilización de la técnica de pre-test, post-test y post-test postergado, pudieron concluir que los dos grupos que recibieron devolución docente emplearon los artículos con mayor precisión que el grupo control en los post-tests. Por otro lado, no encontraron diferencias significativas en la efectividad de la devolución docente focalizada y no focalizada. Muñoz Muñoz (2017) investigó la efectividad de la devolución docente escrita directa e indirecta, focalizada y no focalizada, para ayudar a los estudiantes de educación secundaria a adquirir mayor precisión en el uso del morfema -s en la tercera persona singular en presente simple. Este estudio fue llevado a cabo en un contexto muy similar al del presente proyecto; fue realizado en un contexto de enseñanza de ILE, en una escuela secundaria de Chile. Sus conclusiones indicaron que la devolución docente indirecta y focalizada ayudó a los estudiantes a mejorar su uso del morfema -s en la tercera persona singular en sus composiciones. Sin embargo, Muñoz Muñoz aclara que es necesario realizar nuevos estudios con muestras más numerosas que incluyan un grupo control en sus diseños metodológicos. Esta observación surge a raíz de que —en su trabajotodos los participantes recibieron devolución docente indirecta focalizada. Por lo tanto, a la fecha, no se registran datos que sirvan para comparar estos hallazgos con otros en los que se analicen diferentes tipos de devolución o con su ausencia. 
Berardo, E. \& Luchini, P. (2021). Percepciones y preferencias de estudiantes de inglés en secundaria sobre la devolución docente escrita. UCMaule, 67, julio-diciembre, 11-33. https://doi.org/10.29035/ ucmaule.61.11

\section{Percepciones de los estudiantes sobre la devolución docente}

Diferentes estudios sobre la percepción de los estudiantes sobre la devolución docente indagan sobre situaciones y contextos específicos. Es decir, buscan conocer acerca de las percepciones de los estudiantes sobre el feedback recibido en un determinado contexto, a veces complementándolo con el análisis de la devolución docente que reciben. Tal es el caso del estudio de Montgomery y Baker (2014), en el que las autoras llegaron a la conclusión de que la percepción de los estudiantes acerca del feedback recibido está estrechamente relacionada con los datos obtenidos mediante el análisis de la devolución recibida. En este mismo estudio también se analizaron las percepciones de los docentes sobre sus propias prácticas. Los hallazgos de los datos provenientes de los docentes revelaron que existen discrepancias entre las percepciones de los docentes y lo que realmente hacen en la práctica.

Amrhein y Nassaji (2010) investigaron las percepciones de docentes y estudiantes de inglés como segunda lengua sobre el feedback en general y las compararon y contrastaron entre sí. En sus resultados observaron que, por un lado, los docentes intentaron hacer una selección consciente del tipo de errores a considerar sobre el cual hacer sus devoluciones y cuáles podrían ser ignorados para evitar sobrecargar cognitivamente a sus estudiantes. Por su parte, los estudiantes sugirieron que la utilidad y validez del feedback estaba estrictamente relacionada con a la cantidad de devolución recibida. Tanto estudiantes como docentes manifestaron tener preferencia por resaltar el mismo error varias veces en un mismo texto. Esta práctica se contradice con lo que algunos expertos argumentan. Al respecto, estos investigadores sostienen que resaltar un error al inicio del texto e instar a los alumnos a que, en casos sucesivos, lo corrijan por cuenta propia fomenta el desarrollo de la auto-eficacia y el aprendizaje autónomo. En relación al desarrollo de la autonomía, los docentes de este estudio mostraron preocupación por promoverla a través del tipo de feedback que administraron. Si bien sugirieron estar a favor de la devolución menos explícita, sus estudiantes, en sus comentarios, favorecieron el formato explícito de la devolución, porque su forma directa, contribuye a mostrar la transparencia del mensaje transmitido por sus docentes.

Conocer las percepciones de los estudiantes sobre las devoluciones es un recurso muy valioso para el investigador, sobre todo si se piensa en el feedback como una instancia de interacción y diálogo entre estudiantes y docentes. Si bien los docentes deben tomar decisiones metodológicas basadas en su experiencia profesional, su 
Berardo, E. \& Luchini, P. (2021). Percepciones y preferencias de estudiantes de inglés en secundaria sobre la devolución docente escrita. UCMaule, 61, julio-diciembre, 11-33. https://doi.org/10.29035/ ucmaule.61.11

formación académica y el conocimiento generado a partir de investigaciones propias y ajenas, es importante tener en cuenta la voz de los estudiantes. La voz de los estudiantes constituye un objetivo esencial de aprendizaje que demuestra el desarrollo del concepto de originalidad. Adquirir dominio sobre su voz implica desarrollar una mayor capacidad para aprender y comprender un determinado tema, alentando el aprendizaje autodirigido. El presente estudio tiene como propósito evaluar la efectividad del feedback y para ello suma esta dimensión cualitativa, porque es importante para la toma de decisiones metodológicas de intervención que consideran variables tanto afectivas como cognitivas.

\section{Objetivo}

El objetivo del presente estudio es analizar las percepciones sobre la devolución docente de estudiantes con diferentes niveles de competencia lingüística en inglés con el fin de identificar qué consideran una buena devolución docente. Para ello, se tendrá en cuenta tanto la cantidad como el tipo de devolución. Asimismo, se determinará si el nivel de manejo de la L2 es una variable a considerar cuando se analizan las percepciones de los estudiantes con respecto a las devoluciones que reciben.

\section{MEOTODOLOGÍA}

\section{Participantes}

Participaron en este estudio 62 estudiantes de entre 15 y 17 años ( $M=16$ años) que cursaban la secundaria superior en instituciones privadas de la ciudad de Mar del Plata, Provincia de Buenos Aires, Argentina. Todos ellos cursaban inglés como asignatura en el colegio, con una carga horaria de 6 horas semanales. Según su nivel de competencia lingüística en inglés, se los dividió en dos grupos: Grupo 1: A2-B1 y Grupo 2: C1-C2 (según lo indica el Marco Común Europeo de Referencia [MCER]).

\section{Procedimiento}

Se realizó una adaptación del cuestionario diseñado por Amrhein y Nassaji (2010), en el que los autores incluyeron preguntas de instrumentos de otras investigaciones, previamente piloteadas y modificadas. Este mismo instrumento fue adaptado, traducido al español y empleado por López Casoli y Berardo (2018) en un estudio propio en el que evaluaron las percepciones docentes. Con base en esta fase experimental, en el presente trabajo, se alteró el orden y la redacción de algunas preguntas, se agregaron otras abiertas y cerradas y se incluyó una escala del tipo Likert. 
Berardo, E. \& Luchini, P. (2021). Percepciones y preferencias de estudiantes de inglés en secundaria sobre la devolución docente escrita. UCMaule, 67, julio-diciembre, 11-33. https://doi.org/10.29035/ ucmaule.61.11

Se empleó la plataforma Formularios de Google y se compartió el enlace al cuestionario con los diferentes grupos de estudiantes. La recolección de datos fue realizada durante el mes de septiembre de 2020 .

Los datos obtenidos se analizaron y se volcaron en tablas para facilitar la comparación de los resultados. En el caso de las preguntas cerradas, se consideró la cantidad de veces que se eligió cada opción de respuesta para luego determinar qué porcentaje del total de respuestas representaba cada alternativa. Para analizar los resultados de las preguntas en la que se utilizó la escala de Likert se calculó el promedio de valoración para cada alternativa en cada grupo, y luego se realizó una prueba T para determinar si los resultados fueron estadísticamente significativos. Las respuestas a las preguntas abiertas se analizaron mediante la creación de categorías, estableciendo como índice de valoración la frecuencia con la que se mencionó cada concepto.

\section{RESULTADOS}

\section{Cantidad de feedback}

La primera pregunta del cuestionario apuntaba a indagar sobre las preferencias de los estudiantes en cuanto a la cantidad de feedback recibido. Se les solicitó a los estudiantes que indiquen la afirmación o las afirmaciones con las que estaban de acuerdo. Como se puede observar en la Tabla 1, no se registraron diferencias significativas entre las elecciones de cada grupo de estudiantes.

Para ambos grupos de estudiantes se estableció el mismo orden de preferencia de las opciones según el porcentaje en que aparece cada una de ellas dentro del total de respuestas (Tabla 1). Una amplia mayoría prefiere que el docente resalte todos los errores (Grupo 1: 86,8\%, Grupo 2: 83,3\%), coincidiendo con los resultados expuestos por Amrhein y Nassaji (2010). La segunda opción más elegida fue aquella en la que el docente sólo marcó los errores que interferían en la comunicación de las ideas (Grupo 1: 18,4\%, Grupo 2: 16,7\%). Se observa aquí una importante diferencia entre los datos provenientes del estudio realizado por Amrhein y Nassaji y los del presente trabajo. Siguiendo el orden de preferencias, aparecen dos afirmaciones muy similares entre sí: el docente debe marcar los errores más graves y dejar algunos menos graves sin marcar (Grupo 1: 15,8\%, Grupo 2: 16,7\%) o el docente debe señalar la mayoría de los errores, pero no necesariamente todos (Grupo 1: 5,3\%, Grupo 2: 8,3\%). En este último caso, se deja abierta la posibilidad de elegir otros criterios de selección que no sean necesariamente la gravedad de los errores. Por último, sólo en el Grupo 1 se marcó la opción en la que 
Berardo, E. \& Luchini, P. (2021). Percepciones y preferencias de estudiantes de inglés en secundaria sobre la devolución docente escrita. UCMaule, 61, julio-diciembre, 11-33. https://doi.org/10.29035/ ucmaule.61.11

el docente debería marcar sólo alguno de los errores más graves. Ningún participante consideró útil que el docente solamente haga comentarios relacionados al contenido de las composiciones de los estudiantes. Este resultado coincide con los datos relevados provenientes de las preguntas subsiguientes.

Tabla 1: Respuestas relacionadas a la cantidad de feedback preferido

\begin{tabular}{l|l|l|l|}
\hline \multicolumn{1}{|c|}{ Afirmaciones } & Grupo 1: A2-B1 & Grupo 2: C1-C2 \\
\hline
\end{tabular}

En la segunda pregunta se solicitó a los estudiantes que justifiquen su elección en la pregunta 1. El análisis se realizó teniendo en cuenta las categorías de análisis provenientes de las ideas mencionadas en los comentarios. A continuación, se detallan las categorías de análisis junto con algunos ejemplos citados de las respuestas de los participantes:

$\neq$ Responsabilidad del docente: "La función de un profesor es acompañar al alumno y ayudarlo en todos sus errores, no deberían haber cosas que se pasen por alto" (sic).

$\neq$ Herramienta de aprendizaje: "Al corregir todos los errores los alumnos podrán tener un mejor rendimiento y aprendizaje."

\# Carga cognitiva y/o afectiva: "Marcar todos los errores es importante, pero si son muchos, cuesta diferenciar los más importantes de los que son por ejemplo una falta de ortografía"; "Por otro lado creo que si un alumno tiene muchos errores y se marcan absolutamente todos, quizás podría tender a desilusionarse y desganarse, por lo que sería bueno comenzar con los más generales y luego seguir con los más específicos". 
Berardo, E. \& Luchini, P. (2021). Percepciones y preferencias de estudiantes de inglés en secundaria sobre la devolución docente escrita. UCMaule, 67, julio-diciembre, 11-33. https://doi.org/10.29035/ ucmaule.61.11

$\neq \quad$ Expectativas del estudiante: "Al mandar un trabajo para corregir lo que se espera es que se corrija todo".

$\neq \quad$ Falta de autonomía del estudiante: "Si no marca todos los errores no sabemos en qué nos equivocamos con lo cual volveríamos a cometer los mismos errores más adelante" (sic).

En la Tabla 2 se presenta la frecuencia con la que se mencionó cada categoría en cada grupo. La cantidad total de ideas es igual en cada grupo porque, en primer lugar, no todos los participantes justificaron su respuesta, y, en otros casos, en una misma justificación se encontró más de una categoría.

Tabla 2: Justificaciones sobre la cantidad de feedback preferido

\begin{tabular}{|c|c|c|c|c|}
\hline \multirow{2}{*}{ Categorías } & \multicolumn{2}{|c|}{ Grupo 1: A2-B1 } & \multicolumn{2}{|c|}{ Grupo 2: $\mathrm{Cl}-\mathrm{C}_{2}$} \\
\hline & $\mathbf{N}$ & $\%$ & $\mathbf{N}$ & $\%$ \\
\hline Responsabilidad del docente & 2 & 6,7 & 2 & 6,7 \\
\hline Herramienta de aprendizaje & 14 & 46,6 & 12 & 40 \\
\hline Carga cognitiva y/o afectiva & 2 & 6,7 & 4 & 13,3 \\
\hline Expectativas del estudiante & 2 & 6,7 & 3 & 10 \\
\hline Falta de autonomía del estudiante & 10 & 33,3 & 9 & 30 \\
\hline TOTAL & 30 & 100 & 30 & 100 \\
\hline
\end{tabular}

Nuevamente, se encontraron resultados muy similares en ambos grupos. Como se observó en la Tabla 1, los estudiantes de ambos grupos prefirieron recibir la mayor cantidad de feedback posible y, en su mayoría, justificaron su preferencia resaltando el valor del feedback como herramienta de aprendizaje. En sus comentarios, los estudiantes explicaron que mientras másfeedback reciban, tendrán más oportunidades de aprender. Estos hallazgos coinciden con los datos obtenidos por Amrhein y Nassaji (2010). En segundo lugar, es necesario destacar la falta de autonomía de los estudiantes para reconocer y corregir sus propios errores. El constructo de la autogestión del aprendizaje/regulación del aprendizaje aparece recurrentemente en estudios donde se aborda la temática de la devolución docente, independientemente del nivel de manejo de la lengua de los estudiantes. Pareciera ser que el nivel de competencia lingüística en L2 no está necesariamente relacionado con la capacidad de auto-monitoreo de sus propias producciones. Ambos grupos resaltaron las expectativas de los estudiantes y la 
Berardo, E. \& Luchini, P. (2021). Percepciones y preferencias de estudiantes de inglés en secundaria sobre la devolución docente escrita. UCMaule, 67, julio-diciembre, 11-33. https://doi.org/10.29035/ ucmaule.61.11

responsabilidad y/o rol del docente. Sin embargo, el grupo B (el más avanzado) destacó el rol que cumple la carga cognitiva y/o afectiva, mencionando estos aspectos con más frecuencia. En este último punto, se observa una diferencia interesante con respecto los resultados provenientes del estudio realizado por Amrhein y Nassaji (2010). Si bien sus estudiantes no mencionaron la carga cognitiva y/o afectiva en sus comentarios, los docentes resaltaron este aspecto. Posiblemente, las diferencias encontradas entre su estudio y el presente trabajo sean el resultado de cuestiones netamente culturales, propias de cada región.

En otra de las preguntas se planteó un caso hipotético en el que un mismo error apareció más de una vez en el mismo texto. Se les preguntó a los participantes si desearían que el docente se lo marcara cada vez que apareciera. En la Tabla 3 se observa que, en ambos grupos, una amplia mayoría prefiere que se marquen los errores repetidos. Esta respuesta podría tener relación con lo expresado en la primera pregunta sobre la preferencia por recibir feedback sobre todos los errores que aparecen en el texto evaluado.

Tabla 3: Respuesta de los participantes a si desearían que el docente marque un error repetido

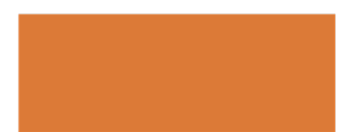

Sí

No

TOTAL

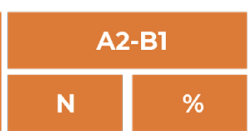

30

8

38

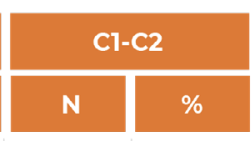

83,8

16,7

100

Al leer los comentarios que explicaban su elección, se encontró poca diversidad en las ideas de ambos grupos. En la Tabla 4 se observan las categorías mencionadas en las respuestas de los participantes que eligieron recibir comentarios acerca de cada uno de sus errores, aun cuando el error era recurrente en el mismo texto.

Tabla 4: Justificaciones a la elección de recibir correcciones cada vez que un error se repita en el mismo texto 
Berardo, E. \& Luchini, P. (2021). Percepciones y preferencias de estudiantes de inglés en secundaria sobre la devolución docente escrita. UCMaule, 67, julio-diciembre, 11-33. https://doi.org/10.29035/ ucmaule.61.11

\begin{tabular}{|c|c|c|c|c|}
\hline \multirow{2}{*}{ Categorías } & \multicolumn{2}{|c|}{ Grupo 1: A2-B1 } & \multicolumn{2}{|c|}{ Grupo 2: C1-C2 } \\
\hline & $\mathbf{N}$ & $\%$ & $\mathbf{N}$ & $\%$ \\
\hline Falta de autonomía del estudiante & 23 & 79,3 & 9 & 47.4 \\
\hline Feedback como ayuda visual & 6 & 20,7 & 6 & 31,6 \\
\hline Diferencias contextuales & 0 & o & 4 & 21 \\
\hline TOTAL & 29 & 100 & 19 & 100 \\
\hline
\end{tabular}

La referencia a la falta de autonomía del estudiante para identificar sus propios errores fue la idea más recurrente para explicar por qué, el marcar los errores sólo una vez, no sería suficiente, con un porcentaje más alto en el grupo 1 que en el grupo 2. En algunos casos, algunos estudiantes agregaron que, quizás, sería interesante que los docentes marcaran todos los errores al comienzo del proceso de aprendizaje, pero que de a poco vayan dejando de hacerlo para que los estudiantes puedan desarrollar su autonomía progresivamente. En segundo lugar, ambos grupos de estudiantes destacaron que marcar los errores repetidos tiene un efecto visual más claro. Esta ayuda visual hace que los estudiantes entiendan mejor que es un error recurrente y, a su vez, ayuda a que le presten más atención y recuerden los comentarios del docente con mayor facilidad. Algunos de estos participantes, incluso, sugirieron que una vez que el error es corregido en primera instancia, luego, el docente podría utilizar feedback implícito ("Creo que en la primera vez que aparece podrían marcarlo y explicar el por qué está mal, y el resto de las veces solo resaltarlo").

Por otro lado, otro grupo de estudiantes indicó que los errores que se repiten recurrentemente en el mismo texto no deberían marcarse, explicando sus razones. Asimismo, en ambos grupos se hizo mención de que luego de haber visto una respuesta patrón por primera vez en un texto, los estudiantes deberían ser capaces de identificar cada error por cuenta propia. Dentro del grupo 1, otros comentarios apelaron a la practicidad, por parte del docente, de marcar los errores solo una vez y, de este modo, alivianar su tarea de corrección. Por su parte, otro comentario resaltó la poca utilidad de tener que volver a corregir el mismo error dentro del mismo texto.

\section{Diferentes tipos de feedback}

Para averiguar las percepciones de los estudiantes sobre la utilidad de cada tipo de feedback, se utilizó una oración que contenía un error gramatical (uso del pasado 
Berardo, E. \& Luchini, P. (2021). Percepciones y preferencias de estudiantes de inglés en secundaria sobre la devolución docente escrita. UCMaule, 61, julio-diciembre, 11-33. https://doi.org/10.29035/ ucmaule.61.11

perfecto en lugar del pasado simple) como ejemplo y se les presentaron diferentes formas de marcar ese error. Los participantes debían valorar la utilidad de cada tipo de corrección mediante una escala de tipo Likert con una progresión de 1 a 5, nominalizada de la siguiente manera: 1=para nada útil, 2=no muy útil, 3=es indistinto, 4= bastante útil, 5=muy útil. En la Tabla 5 se observan las medias obtenidas para cada ítem en cada grupo y los valores de t y p según la prueba T para muestras independientes.

Tabla 5: Comparación de la media de la valoración de la utilidad de cada tipo de feedback

\begin{tabular}{|c|c|c|c|c|}
\hline Tipo de Feedback & $\begin{array}{l}\text { Media Grupo } 1 \\
\text { (A2-B1) }\end{array}$ & $\begin{array}{l}\text { Media Grupo } 2 \\
\quad(\mathrm{Cl}-\mathrm{C} 2)\end{array}$ & $\begin{array}{c}\text { Valor } \\
t\end{array}$ & $\begin{array}{c}\text { Valor } \\
p\end{array}$ \\
\hline a. Usar metalenguaje para definir el tipo de error & 3.63 & 3.91 & $-0,955$ & .343 \\
\hline b. Usar una pregunta & 2.73 & 3.37 & $-2,189$ & .32 \\
\hline c. Usar un imperativo & 3.00 & 3.87 & -3.982 & .000 \\
\hline d. Escribir la forma correcta de corregir el error & 3,63 & 3,45 & 0,580 & .564 \\
\hline c. Escribir una afirmación explicando cl tipo de error & 3,63 & 3,66 & $-0,137$ &, 892 \\
\hline f. Usar una exclamación & 2,94 & 3,20 & $-0,784$ & 436 \\
\hline $\begin{array}{l}\text { g. Señalar la existencia del error sin indicar cuál es ni } \\
\text { cómo solucionarlo }\end{array}$ & 2,10 & 2,20 & $-0,390$ & .698 \\
\hline h. No marcar el error & 1,18 & 1,04 & 1.438 & .156 \\
\hline
\end{tabular}

El análisis de la valoración de cada tipo de corrección muestra pocas diferencias estadísticamente significativas entre ambos grupos. Usar metalenguaje, escribir la forma correcta y escribir una afirmación explicando el tipo de error tuvieron una valoración media que supera el punto de valoración neutral ("es indistinto") en ambos grupos, indicando que, a estudiantes de ambos niveles, les resultaría útil ese tipo de corrección. El uso de exclamaciones en la corrección fue valorado de forma más neutral en ambos grupos. Por otro lado, señalar sólo la existencia y la ubicación del error, o no marcarlo de ninguna manera obtuvieron las valoraciones más bajas, coincidiendo con las respuestas ya mencionadas en el análisis de otros incisos de la encuesta.

Se observaron diferencias estadísticamente significativas entre los dos grupos para las preguntas y los imperativos. El grupo 1 dio una valoración significativamente más baja 
Berardo, E. \& Luchini, P. (2021). Percepciones y preferencias de estudiantes de inglés en secundaria sobre la devolución docente escrita. UCMaule, 67, julio-diciembre, 11-33. https://doi.org/10.29035/ ucmaule.61.11

al uso de preguntas ( $M=2,73, S D=1,10)$ que el grupo $2(M=3,37, S D=1,13), t(60)=-2,189$, $p=, 03)$. Esta diferencia podría estar justificada por el hecho de que, de los tipos de comentarios de feedback incluidos en el cuestionario, las preguntas que interpelan a los estudiantes resultaron ser las menos explícitas, alentándolos no sólo a encontrar la solución al problema señalado, sino a determinar de qué tipo de error se trata. Quizás, los estudiantes más avanzados valoren este desafío como una instancia más útil, porque sienten que cuentan con más herramientas para enfrentarlo.

En cuanto al uso de imperativos, el grupo 1 también mostró una diferencia significativamente más baja $(M=3,00, S D=0,80)$ que el grupo $2(M=3,87, S D=0,89), t$ $(60)=-3,982, p=, 00)$. En varias ocasiones, las diferentes valoraciones sobre este tipo de comentario se asocian a variables individuales relacionadas a lo motivacional, lo afectivo e, incluso, lo cultural (López Casoli y Berardo, 2016). En este caso, el Grupo 1 no consideró que fueran especialmente útiles o poco útiles, mientras que el Grupo 2 los valoró con una media más cercana al "bastante útil". Quizás, estos estudiantes más avanzados contaban con mayor seguridad, dado su alto nivel de competencia lingüística en L2 (recordemos que, al momento de la recolección de datos, los estudiantes de ambos grupos tenían la misma edad y habían estudiado inglés durante la misma cantidad de años). Este nivel alto de seguridad puede lograr mitigar los efectos afectivos del uso de un comentario que suele ser recibido como intimidante (Sotoudehnama \& Molavi, 2014).

Inmediatamente después de la pregunta en la que se solicitaba a los participantes valorar la utilidad de cada tipo de corrección, se incluyó un espacio para que escribieran una breve justificación. En estos comentarios se encontraron indicaciones de lo que los estudiantes consideraron "buen o mal feedback". Los criterios de evaluación del feedback que los participantes mencionaron están representados por las categorías que se detallan en la Tabla 6. A continuación, también se incluyen ejemplos para cada categoría analizada:

Claridad/evitar ambigüedad: "Personalmente, me resulta más fácil que me marquen dónde está el error, a que no pongan nada, porque eso sino pienso que está bien y no es así."

Desarrollo lingüístico (aprendizaje a largo plazo a partir de las correcciones):

"Took capaz no está tan bueno, porque lo cambio automáticamente y me olvido de mi error".

Desarrollo de la autonomía: "Las correcciones que me parecieron más útiles fueron aquellas que no dan la respuesta directa ya que permiten que 
Berardo, E. \& Luchini, P. (2021). Percepciones y preferencias de estudiantes de inglés en secundaria sobre la devolución docente escrita. UCMaule, 67, julio-diciembre, 11-33. https://doi.org/10.29035/ ucmaule.61.11

el alumno piense por sí mismo, sabiendo que tiene los conocimientos previos, y que pueda encontrar más de una respuesta correcta".

Falta de autonomía de los estudiantes: "Obviamente, para mí lo que yo escribí está bien, entonces necesito que la corrección me explique mi error".

Efectos en lo afectivo/en la motivación: "Aunque cumpla la misma función que el anterior me parece que uno como alumno se lo toma distinto, suena más feo. Es la peor forma de expresarlo. Creo que así ni siquiera me darían ganas de buscar la forma de hacerlo bien."

Tabla 6: Justificaciones sobre el tipo de feedback preferido

\begin{tabular}{|c|c|c|c|c|}
\hline \multirow{2}{*}{ Categorías } & \multicolumn{2}{|c|}{ Grupo 1: A2-B1 } & \multicolumn{2}{|c|}{ Grupo 2: C1-C2 } \\
\hline & $\mathbf{N}$ & $\%$ & $\mathbf{N}$ & $\%$ \\
\hline Claridad / evitar ambigüedad & 5 & 31,3 & 6 & 24 \\
\hline Desarrollo lingüístico & 3 & 18,7 & 2 & 8 \\
\hline Desarrollo de la autonomía & 2 & 12,5 & 11 & 44 \\
\hline Falta de autonomía de los estudiantes & 4 & 25 & 2 & 8 \\
\hline Efectos en lo afectivo / en la motivación & 2 & 12,5 & 4 & 16 \\
\hline TOTAL & 16 & 100 & 25 & 100 \\
\hline
\end{tabular}

El grupo de estudiantes nivel A2-B1 mencionó, con mayor frecuencia, la necesidad de claridad en las correcciones brindadas por los docentes. Esto va de la mano con la falta de autonomía de los estudiantes, criterio que le sigue en el orden de mayor a menor frecuencia. Nuevamente, vuelve a aparecer esta idea en este mismo grupo: los estudiantes necesitan la ayuda del docente para poder editar sus textos, destacando que esa ayuda debe ser lo más clara posible. Anteriormente, habían mencionado las correcciones de los docentes como una ayuda visual al momento de analizar sus producciones y editarlas, y ahora vuelven a caracterizar el feedback como un sistema de orientación (Burke \& Pieterick, 2010). Sin embargo, el rol del feedback en el desarrollo de la autonomía fue mencionado sólo en dos comentarios.

Por otro lado, si bien el grupo de participantes de nivel C1-C2 también mencionó la falta de autonomía de los estudiantes, lo hizo con menor frecuencia que el otro grupo y se enfocó en destacar el potencial desarrollo de la autonomía a través de la búsqueda de la forma correcta. Esa idea fue la que apareció con mayor frecuencia en las justificaciones, seguida por la importancia de la claridad de los comentarios. 
Berardo, E. \& Luchini, P. (2021). Percepciones y preferencias de estudiantes de inglés en secundaria sobre la devolución docente escrita. UCMaule, 67, julio-diciembre, 11-33. https://doi.org/10.29035/ ucmaule.61.11

Los efectos que los comentarios de los docentes puedan producir a nivel afectivo y motivacional fueron tenidos en cuenta con mayor frecuencia, nuevamente, en el grupo de estudiantes más avanzados.

\section{CONCLUSIONES Y DISCUSIÓN}

Una de las reflexiones centrales que se desprende del análisis de este conjunto de datos es que, sin dudas, los estudiantes valoran el feedback que reciben y lo perciben como una herramienta útil de aprendizaje, apoyando lo argumentado por Ferris (2016). Reconocen que estos comentarios contribuyen al desarrollo de su interlengua, ya sea ayudándolos a enfocar su atención en ciertos aspectos que deben trabajar o proveyéndoles información sobre el uso de la lengua (input). Estos resultados están alineados con las perspectivas teóricas sobre motivación y la evidencia empírica que apoya la efectividad de la devolución docente (Ellis et al., 2008).

A través de las justificaciones que volcaron los participantes en el cuestionario se evidencia un sistema de conceptos o categorías que estructuran las percepciones que tienen los estudiantes sobre el feedback. El concepto de autonomía está muy presente en sus reflexiones. Por un lado, reconocen, en muchos casos, no contar con el grado de autonomía necesario para revisar sus textos sin ayuda, o para corregir sus errores sin haber recibido las respuestas correctas, sobre todo los estudiantes de niveles más bajos. Por otro lado, los estudiantes más avanzados aprecian que se les otorgue la oportunidad de buscar, por cuenta propia, la forma de mejorar sus textos y reconocen que esto, poco a poco, contribuye a desarrollar su autonomía. Este mismo grupo de participantes también se mostró más preocupado por la carga cognitiva o afectiva al recibir grandes cantidades de feedbacky, quizás, se deba a que, también, sus producciones son extensas y se espera que manipulen estructuras léxico-gramaticales más complejas. En un estudio realizado en la Universidad Nacional de Mar del Plata, con estudiantes del mismo nivel de manejo de la lengua (C1-C2), que indagaba sobre la efectividad de la devolución focalizada y no focalizada, Machado, Lucas y Berardo (2015) observaron el efecto adverso de sobrecargar el texto al hacer comentarios de forma irrestricta. Los participantes que habían recibido feedback no sostuvieron el mismo nivel de participación que los que habían recibido feedback focalizado, mostrando una disminución en su nivel de motivación.

En ambos grupos, los participantes reflexionaron sobre el rol y la responsabilidad del docente frente a la corrección. Le otorgaron un papel de facilitador del proceso 
Berardo, E. \& Luchini, P. (2021). Percepciones y preferencias de estudiantes de inglés en secundaria sobre la devolución docente escrita. UCMaule, 61, julio-diciembre, 11-33. https://doi.org/10.29035/ ucmaule.61.11

de escritura y de aprendizaje al acompañarlos en la edición de sus textos, y también dejaron ver, a través de sus reflexiones, que existen ciertas expectativas sobre lo que el docente debe y no debe hacer al momento de asistirlos.

Cuando fueron indagados acerca de la utilidad de diferentes métodos de corrección, ambos grupos demostraron una marcada preferencia por las formas más explícitas de devolución. Según Gass (2018), los distintos métodos incluidos en el cuestionario pueden clasificarse en base a dos ejes: explícito o implícito y directo o indirecto. Los imperativos, el uso de metalenguaje, las exclamaciones que indican el tipo de error, los comentarios con explicaciones y las preguntas tienen cierto grado de explicitud, aunque no indiquen una alternativa para reescribir la sección del texto que contiene el error, que sería la opción más directa. Por lo tanto, aun así guían al estudiante en una dirección más clara que la simple localización de un error, aunque, por supuesto, existe el riesgo de que el estudiante no encuentre la forma de solucionar el problema.

La diferencia más marcada y estadísticamente significativa, entre los dos grupos de participantes, se encontró al analizar la valoración media de la utilidad del uso de preguntas e imperativos. Las preguntas ayudan a problematizar el proceso de reescritura, pero para los estudiantes de niveles más bajos parecen ser menos claras. Manifestaron necesitar instrucciones que no sean ambiguas. Los estudiantes más avanzados las valoraron con el mismo grado de utilidad que los tipos de comentarios más explícitos.

La valoración del uso de imperativos también fue juzgada más favorablemente por los estudiantes más avanzados, aunque varios de ellos los percibieron descorteses. Varias investigaciones empíricas sostienen que el uso de imperativos es conducente a mejoras en las producciones de los estudiantes a corto plazo (Iravani et al., 2014; López Casoli y Berardo, 2016; Sugita, 2006; Rashtchi \& Mirshahidi, 2011), mientras otras sostienen lo contrario (Ferris et al., 1997; Sotoudehnama \& Molavi, 2014; Tajik et al., 2016). La diversidad de los contextos en las que se realizaron estos estudios parece indicar que los resultados disímiles se deben, principalmente, a diferencias culturales y a otras diferencias individuales dentro de cada grupo de participantes.

Una de las implicancias pedagógicas de estos resultados es que, dado que los estudiantes valoran la claridad de los comentarios, es importante explicitar (y, por qué no, consensuar) las estrategias del docente a la hora de brindar feedback. Como se puede observar en los comentarios, algunas omisiones que los docentes hacen por practicidad, o guiados por algún principio metodológico (preferencia por el feedback 
Berardo, E. \& Luchini, P. (2021). Percepciones y preferencias de estudiantes de inglés en secundaria sobre la devolución docente escrita. UCMaule, 67, julio-diciembre, 11-33. https://doi.org/10.29035/ ucmaule.61.11

selectivo, atención a la carga cognitiva, etc.) a veces son interpretados como falta de atención al detalle por los estudiantes. Esta actitud de los docentes puede frustrar a los estudiantes al ver correcciones nuevas sobre esos mismos aspectos en borradores subsiguientes. Según Mochizuki (2017, en Ellis et al., 2008), es necesario "equipar a los estudiantes con las estrategias necesarias para dar y recibir feedback" y "tratar las relaciones de poder que suprimen la participación de los estudiantes en dar y recibir feedback" (p. 115).

Así como en el caso de los estudios sobre el uso de los imperativos, la investigación y reflexión sobre el feedback, en general, va por dos caminos diferentes, que a veces se fusionan: las percepciones de los actores involucrados (docentes y estudiantes) y la realidad (los métodos que realmente se utilizan, más allá de las intenciones del docente, y la efectividad de los mismos). Es necesario que se investiguen ambas áreas para poder guiar a los docentes a tomar mayor consciencia acerca de las reflexiones de sus estudiantes para tratar de cumplir con sus expectativas y lograr sostener su motivación. A la vez, este conocimiento les permitirá elegir los métodos más eficaces para guiar a sus estudiantes con el fin de ayudarlos a alcanzar los objetivos propuestos.

\section{REFERENCIAS BIBLIOGRÁFICAS}

Amrhein, H. R. \& Nassaji, H. (2010). Written corrective feedback: what do students and teachers prefer and why. Canadian Journal of Applied Linguistics, 13(2), 95127. https://journals.lib.unb.ca/index.php/CJAL/article/view/19886

Beckner, C., Blythe, R., Bybee, J., Christiansen, M. H., Croft, W., Ellis, N. C., Holland, J., Ke, J., Larsen-Freeman, D. \& Schoenemann, T. (2009). Language is a complex adaptive system: Position paper. Language Learning, 59, 1-26. https://doi. org/10.1111/j.1467-9922.2009.00533.x

Bitchener, J. (2008). Evidence in support of written corrective feedback. Journal of Second Language Writing, 17, 102-118. https://doi.org/10.1016/j.js/w.2007.11.004

Bitchener, J. (2012). Written corrective feedback for L2 development: Current knowledge and future research. TESOL Quarterly, 46(4), 855-860. http://www.jstor. org/stable/43267894

Burke, D. \& Pieterick, J. (2010). Giving students effective written feedback. Open University Press. 
Berardo, E. \& Luchini, P. (2021). Percepciones y preferencias de estudiantes de inglés en secundaria sobre la devolución docente escrita. UCMaule, 67, julio-diciembre, 11-33. https://doi.org/10.29035/ ucmaule.61.11

Ellis, R. (2009). A typology of written corrective feedback types. ELT Journal, 63(2), 97107. https://doi.org/10.1093/elt/ccn023

Ellis, R. (2015). Grammar teaching for language learning. Babylonia, 2(14), 10-15. http:// babylonia.ch/fileadmin/user_upload/documents/2014-2/Ellis.pdf

Ellis, R., Sheen, Y., Murakami, M. \& Takashima, H. (2008). The Effects of Focused and Unfocused Written Corrective Feedback in an English as a Foreign Language Context. System: An International Journal of Educational Technology and Applied Linguistics, 36(3), 353-371. https://doi.org/10.1016/j.system.2008.02.001

Ferris, D., Pezone, S., Tade, C. \& Tinti, S. (1997). The Case for Grammar Correction in L2 Writing Classes: A Response to Truscott (1996). Journal of Second Language Writing, 8(1), 1-11. https://files.eric.ed.gov/fulltext/EJ844661.pdf

Ferris, D. (1999) "The Case for Grammar Correction in L2 Writing Classes: A Response to Truscott (1996)". Journal of Second Language Writing, 8(1), 1-11. https://doi. org/10.1016/S1060-3743(99)80110-6

Ferris D. (2002). Treatment of Error in Second Language Student Writing. University of Michigan Press.

Ferris, D., Liu, H., Sinha, A., Senna, M. (2012). Written corrective feedback for individual L2 writers. Journal of Second Language Writing, 22(3), 307-329. https://doi. org/10.1016/j.jslw.2012.09.009

Gass, S. (2018). Input, Interaction, and the Second Language Learner. Routledge.

Iravani, H., Hemmati, F. \& Ahmadpoor, F. (2014). The Impact of EFL Teachers' Comment Types on Students' Revision. International Journal of Language Learning and Applied Linguistics World, 7(3), 326-338.

López Casoli, M. y Berardo, E. (2016). El modo imperativo como forma de devolución sobre la escritura en inglés como lengua extranjera. En Castro, A., Agüero, G., Raffo, C., Gonzalez de Gatti, M. M., Romano, M. E., Actas del I Congreso Internacional Lenguas-Migraciones-Culturas. (176-187). http://hdl.handle. net/11086/4567

López Casoli, M. y Berardo, E. (2018) El proceso de devolución sobre la escritura académica en inglés: percepciones de docentes universitarios. Revista Brasileira de Ensino Superior, 4(2), 84-102. https://doi.org/10.18256/2447-3944.2018. v $4 \mathrm{i} 2.2462$ 
Berardo, E. \& Luchini, P. (2021). Percepciones y preferencias de estudiantes de inglés en secundaria sobre la devolución docente escrita. UCMaule, 67, julio-diciembre, 11-33. https://doi.org/10.29035/ ucmaule.61.11

Machado, C., Lucas, S. y Berardo, E. (noviembre, 2015). La influencia del feedback comprensivo y selectivo en el proceso de escritura en Inglés como lengua extranjera. Trabajo presentado en JELENS, XV Jornadas y I Congreso Latinoamericano de Enseñanza de Lenguas Extranjeras en el Nivel Superior, Universidad Nacional de Córdoba, Argentina.

Montgomery, J.L., \& Baker, W. (2007). Teacher-written feedback: Student perceptions, teacher self-assessment, and actual teacher performance. Journal of Second Language Writing, 16, 82-99. https://doi.org/10.1016/j.jslw.2007.04.002

Muñoz Muñoz, B. C. (2017). Contribución del feedback correctivo escrito indirecto en el aprendizaje del morfema -s de verbos en inglés en tercera persona singular, en estudiantes de enseñanza básica. Literatura y Didáctica (Universidad Católica Silva Heríquez), 35, 275-296. http://dx.doi.org/10.4067/S071658112017000100273

Rashtchi, M., \& Mirshahidi, S. (2011). The Primacy of Teacher Imperative Commentaries in the Improvement of Iranian English Majors' Writing Ability. Iranian Journal of Applied Language Studies, I3(1), 119-150. https://ijals.usb.ac.ir/article_87.html

Sotoudehnama, E. \& Molavi, M. (2014). The Effects of Teachers' Written Comment Types and Iranian EFL Learners' Attitudes. The Journal of Asia TEFL, 17(4), 21-51. http://www.asiatefl.org/main/main.php?inx_journals=42\&inx_contents=388\&main=6\&sub=5\&submode=3\&PageMode=JournalView\&s_title=The_Effects_of_Teachers_Written_Comment_Types_and_Iranian_EFL_Learners_Attitudes

Sugita, Y. (2006). The Impact of Teachers' Comment Types on Students' Revisions. ELT Journal, 60(1), 34-40. https://doi.org/10.1093/elt/cci079

Tajik, L., Fakhari, M., Hashamdar, M. \& Zadeh Habib, S. (2016). Three Types of Comments on Content: Teacher vs. Peer Feedback. The Journal of Teaching Language Skills, 7(4), 141-166. https://tesl.shirazu.ac.ir/article_3656.html

Truscott, J. (1996). The Case Against Grammar Correction in L2 Writing Classes. Language Learning, 46(2), 327-369. https://doi.org/10.1111/j.1467-1770.1996.tb01238.x

Truscott, J. (1999). The Case for "The Case Against Grammar Correction in L2 Writing Classes": A Response to Ferris. Journal of Second Language Writing, 8(2), 117122. https://doi.org/10.1016/S1060-3743(99)80124-6 
Berardo, E. \& Luchini, P. (2021). Percepciones y preferencias de estudiantes de inglés en secundaria sobre la devolución docente escrita. UCMaule, 67, julio-diciembre, 11-33. https://doi.org/10.29035/ ucmaule.61.11

Truscott, J. (2004). Evidence and conjecture on the effects of correction: A response to Chandler. Journal of Second Language Writing, 13, 337-343. https://doi.org/10.1016/j.jslw.2004.05.002

Truscott, J. (2007). The effect of error correction on learners' ability to write accurately. Journal of Second Language Writing, 16, 255-272. https://doi.org/10.1016/j. jslw.2007.06.003

\section{APÉNDICE 1 - CUESTIONARIO (ADAPTADO DE AMRHEIN Y NASSAJI, 2010)}

1. Si hay muchos errores para corregir en un texto que escribiste para la clase de inglés, ¿qué pensás que debería hacer tu profesor/a? Podés marcar más de una opción.
debería marcar todos los errores que encuentre
debería marcar todos los errores más "graves" y dejar de lado algunos de los menos "graves"
debería marcar la mayoría de los errores, pero no necesariamente todos debería marcar sólo algunos de los errores más "graves"
debería marcar sólo los errores que interfieran con la comprensión de la idea que quiero expresar
no debería marcar ninguno de los errores y debería sólo hacer comentarios que respondan al contenido del texto, a las ideas que expresé

2. Por favor, justificá brevemente tu respuesta a la pregunta anterior.

3. Diferentes tipos de devolución: Las siguientes oraciones contienen el mismo error y el docente proveyó distintos tipos de devolución en cada una. Para cada oración elegí el número (1-5) que mejor describe la utilidad de la devolución para los estudiantes, siendo 1=para nada útil, 2=no muy útil, 3=es indistinto, 4= bastante útil, 5=muy útil.

4. Por favor, justificá brevemente tus preferencias expresadas en el inciso anterior.

5. Si un error se repite en el mismo texto, ¿te parece útil que te lo marquen cada vez que aparece?

Sí

No

6. ¿Por qué? 
Berardo, E. \& Luchini, P. (2021). Percepciones y preferencias de estudiantes de inglés en secundaria sobre la devolución docente escrita. UCMaule, 61, julio-diciembre, 11-33. https://doi.org/10.29035/ ucmaule.61.11

wrong tense

a- I have taken the children to school yesterday. (usar términos especificos para definir el tipo de error)

Is this the right tense?

b- I have taken the children to school yesterday. (usar una pregunta)

Change tense?

c-I have taken the children to school yesterday. (usar un inmperativo)

Took

d- I have taken the children to school yesterday. (escribir la forma correcta de solucionar el error)

This isn't the right tense of the verb.

e- I have taken the children to school yesterday. (escribir una afirmación explicando el tipo de error)

wrong tense

f I have taken the children to school yesterday. (usar una exclamación)

g- I have taken the children to school yesterday. (señalar dónde hay un error sin decir cúal es)

f- I have taken the children to school yesterday. (no marcar error) 